\title{
The Role of Professional Physical Therapists in Smoking Cessation: A Literature Update
}

Ana Paula Coelho Figueira Freire ${ }^{1 *}$, Dionei Ramos ${ }^{1}$, Bruna Spolador de Alencar Silva ${ }^{1}$, Marceli Rocha Leite1, Juliana Souza Uzeloto ${ }^{1}$, Francis Lopes Pacagnelli ${ }^{2}$ and Ercy Mara Cipulo Ramos ${ }^{1}$

${ }^{1}$ São Paulo State University - Presidente Prudente, São Paulo - Brazil.

${ }^{2}$ Oeste Paulista University - Presidente Prudente, São Paulo - Brazil.

*Corresponding author: Ana Paula Coelho Figueira Freire,Roberto Simonsen, 305 Presidente Prudente, São Paulo, Brazil, Tel: 55 18 988029656; Fax: 5518 32295821; E-mail: anapcff@hotmail.com

Received date: March 31, 2016; Accepted date: March 28, 2017; Published date: April 3, 2017

Copyright: (c) 2017 Freire FAPC. This is an open-access article distributed under the terms of the Creative Commons Attribution License, which permits unrestricted use, distribution, and reproduction in any medium, provided the original author and source are credited.

\begin{abstract}
Objective: To update the literature on the role of the physical therapist in smoking cessation interventions and the effects of smoking in different therapeutic approaches. For this update the Medline, Scielo, Lilacs and Cochrane electronic databases were searched using the following keywords: physical therapy specialty, physical therapists, tobacco use cessation, smoking, national program of tobacco control. The physical therapist presents particularities that enable them a broad role in smoking intervention due to frequent contact with the patient for prolonged periods, generally individually; physical therapy usually takes place several times a week for sessions of one hour or more and for long follow up periods (months or years), characteristics which are quite specific to these professionals.

Conclusion: Physical therapists present particularities and characteristics that enable them to become facilitators in the smoking intervention process, and they can thus act both preventively and as interventionists in this process. It is important that these professionals become aware of their role in smoking cessation and act more clearly in this process, with the goal of optimizing treatment and preventing complications, seeking improvement in the health of their patients.
\end{abstract}

Keywords: Physical therapy specialty; Smoking; Tobacco smoke pollution

\section{Introduction}

It is known that smoking is considered a serious public health issue with high incidence worldwide, leading to high costs to the health system due to tobacco-related diseases [1]. According to the International classification of disease there is a code attributed to mental and behavioural disorders due to use of tobacco and nicotine dependence (ICD-10, F17.2). It is estimated that there are about 1.3 billion smokers in the world, $80 \%$ of these individuals are in low and middle income countries [2].

Brazil has 27.9 million smokers and is considered the second largest producer and the largest tobacco exporter in the world. However, development of strong actions for tobacco control has brought the country recognition as an international leader in this area in recent years. These actions include increasing taxes on cigarettes, publication of research on the effects of smoking on health, intensification of information to consumers through warning labels and replacement of tobacco farming in addition to encouraging cessation interventions [3-5].

In contrast to these implemented actions, Brazil still possesses some of the cheapest cigarettes in the world, with easy access aggravated by a large illegal market for cigarette sales. These factors contribute to a total of 200,000 deaths annually in the country related to smoking as well as high rates of premature death [4-6].
Thus, the need for effective solutions for smoking cessation such as the awareness of health professionals on this subject and encouragement of smoking intervention programs is evident [7]. These programs are designed to help smokers who have a desire to stop smoking, but cannot attain permanent cessation. The most indicated treatment has a specific approach entitled cognitive behavioral therapy, associated with pharmacological therapy directed at smoking cessation $[8,9]$.

Several health professionals are involved in smoking intervention and, if well trained, can act in these specialized programs. The involvement of a multidisciplinary team is critical to attend the smoker. As part of this team, physical therapists play an important role in the process and contribute to complete and specialized care $[10,11]$. Physical therapists are highly-educated, licensed health care professionals who can help patients and can teach patients how to prevent or manage their condition so that they will achieve long-term health benefits.

It is important to emphasize that smoking affects many systems in the body such as the respiratory, cardiovascular, musculoskeletal and neurological systems; and the role of the physical therapist includes clinical practice in all these areas $[10,12]$. Given the above considerations, it is essential to widen the knowledge on the role of the physical therapist in smoking intervention, since smoking can directly influence daily behaviour. Moreover, this activity becomes a speciality area for this professional, expanding and diversifying their activities.

Therefore, the aim of this study was to update the literature on the current smoking scenario, the role of the physical therapist in smoking 
intervention and the effects of cigarettes on the various therapeutic approaches.

\section{Methods}

For this review, the following electronic databases were searched: Medline, Lilacs and Cochrane, using the descriptors of Health Sciences (DeCS): physical therapy, physical therapist, tobacco use cessation, smoking and national tobacco control program.

The inclusion criteria of the sample were based on articles published in Portuguese, English and Spanish, freely available, between 2004 and 2014. Articles were selected based on the analysis of their titles followed by their abstract and those that did not discuss the role of the physical therapist in smoking intervention were excluded from this review, as well as articles that were not related to understanding of the effects of smoking on different areas of physical therapy.

Two investigators independently assessed the articles and evaluated which papers addressed the study's inclusion criteria. In cases of disagreement between the two researchers a third researcher was consulted for the final decision. All selected studies should approach the role of physical therapist in a smoking cessation interception.

A total of 259,282 articles were found, and considering the inclusion criteria, 52 studies were selected for this review (Figure 1).

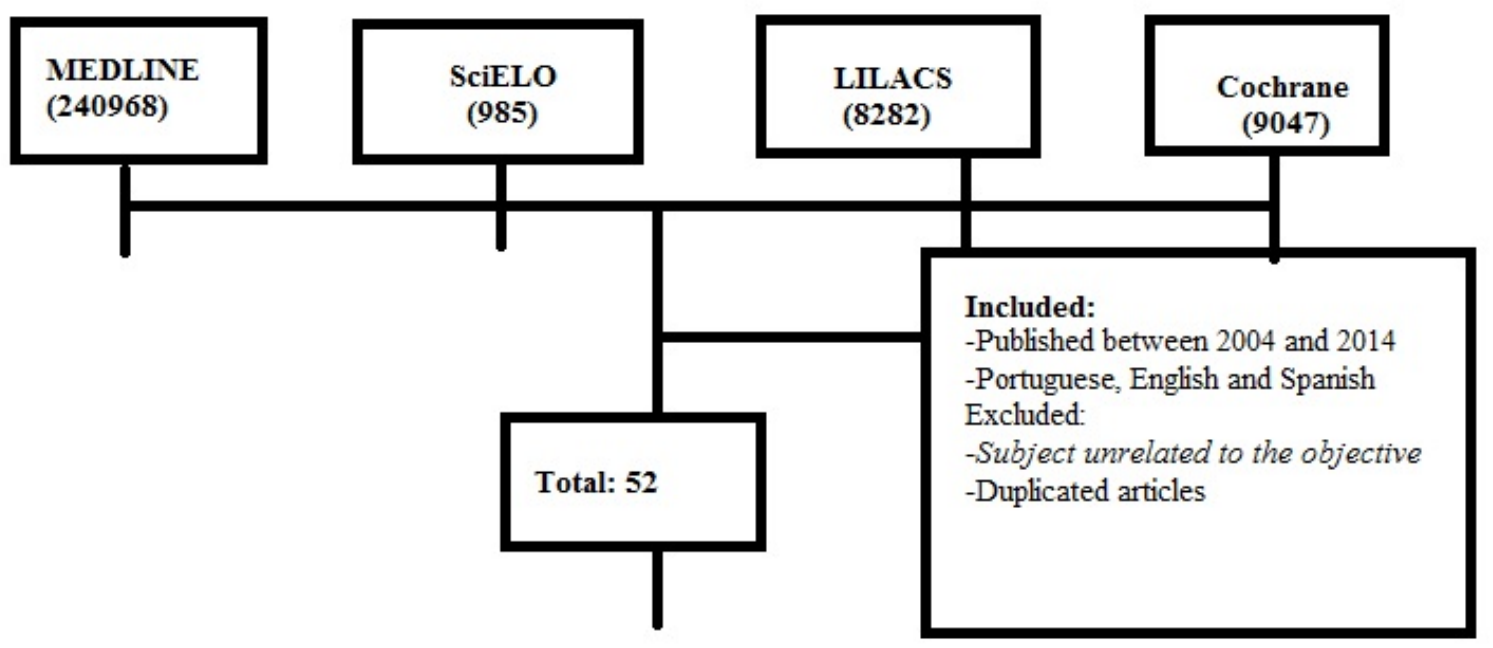

Figure 1: Diagram of the selection of the studies included in the review.

\section{Results and Discussion}

\section{Smoking addiction}

It is often difficult for health professionals to understand why patients continue smoking even after the appearance of severe diseases and impairments related to smoking. Thus, it is important to understand the dependency mechanism experienced by these individuals.

Tobacco addiction is known as a chronic condition that requires specialized intervention [13]. The termination process can involve episodes of relapse that are often not understood by health professionals who are not active in the smoking intervention process. It must be emphasized that tobacco presents a complex dependency mechanism involving chemical, behavioral and psychological components.

\section{Chemical addiction}

The nicotine absorption process occurs in the mouth, lungs and skin and then moves into the blood stream and reaches the brain within seconds. In the brain, nicotine binds to specific structures called nicotinic cholinergic receptors. The binding of nicotine with these receptors promotes certain alterations such as the opening of ion channels.
These channels promote cation influx $\left(\mathrm{Na}^{+}\right.$and $\left.\mathrm{Ca}^{2+}\right)$. The increased permeability of calcium in neurons triggers the release of neurotransmitters that produce rewarding psychoactive effects [14].

Among the released neurotransmitters, dopamine is responsible for a sense of pleasure and well-being considered critical to reinforce the use of nicotine in search of these sensations. At the beginning of their smoking history, the individual develops a process of tolerance to the psychoactive substances in cigarettes, thus, the need for higher doses of these substances increases to achieve the same degree of satisfaction and avoid the many unpleasant symptoms promoted by withdrawal, establishing a process of reinforced substance use.

This mechanism of tolerance may be associated with a significant increase in nicotinic receptors in the brain which demand higher quantities of nicotine. In addition to pharmacological effects, smoking addiction can also result from genetic, social and environmental factors $[14,15]$.

Studies seeking to understand the genetic mechanisms involved in this addiction have demonstrated that there are specific genes that may increase susceptibility to addiction and can be hereditary, however, these studies have important limitations due to the influence of social and environmental factors that are part of this mechanism, such as the influence of friends, family, psychiatric disorders, age and sex [16]. 


\section{Behavioral addiction}

Behavioral addiction is associated with the conditioning to smoke and perform daily activities. In other words, there is an association between smoking and daily activities such as drinking coffee, drinking alcohol, driving, talking on the phone and going to the bathroom. This behavioral sensitization is induced over many years and requires special attention during the cessation intervention process. This behaviour implies that the environment paired with nicotine acquires characteristics of a conditioned stimulus due to the association with the positive reinforcing effects of nicotine [17].

\section{Psychological addiction}

Psychological addiction is characterized by the association between smoking and the manner in which smokers deal with emotions such as sadness, anxiety, depression and even euphoria. In this way, the smoker is conditioned to deal with everyday situations using the cigarette as a tool. In addition, the cigarette acquires an important role in the life of the smoker, becoming not only an object but a daily companion in the routine of the individual; this process is known as the personification of the cigarette [18].

\section{Tobacco Intervention Programs}

Smoking intervention programs are designed to help smokers who want to give up smoking. It is known that $80 \%$ of these individuals have the desire to give up smoking, but only $3 \%$ reach permanent cessation without specialized support [19].

There are several types of approach for the smoker that can be applied by any health professional. These approaches can be performed through minimal counselling ( $<3$ minutes), low intensity counselling (3-10 minutes) and intensive counselling ( $>10$ minutes).

It is important to point out that regardless of the approach used; this type of action promotes an increase in the chance of permanent smoking cessation. Studies demonstrate an estimated rate of abstinence of $10.9 \%$ if the smoker tries to give up smoking alone, versus $13.4 \%$ when undergoing minimum counselling, $16.0 \%$ for low intensity counselling, and $22.1 \%$ when undergoing intensive counselling [20].

Specialized programs for smoking intervention use an intensive approach aiming to act on the three previously mentioned dependent mechanisms, promoting a complete and specific treatment with greater chances of success [8].

To achieve these indices of success, the highest level of scientific evidence points to treatments that include cognitive behavioral therapy, which combines cognitive interventions, with training of behavioral skills in order to modify the positive reinforcement related to smoking, and the use of specialized pharmacological therapy, composed of nicotine replacement therapy (nicotine patches, nicotine gum) and bupropion [8].

These therapies can be structured in groups or individually, with weekly meetings initially and follow-up of six months to one year, relying on the support of many health professionals, such as a psychologist, doctor, nutritionist, dentist, physical educator, occupational therapist and physical therapist. In this intensive approach, the smoker benefits from the multidisciplinary and multiprofessional action and receives guidance and specific care to attempt cessation.
Given the above, it can be inferred that all health professionals should support and encourage smoking cessation through counselling; as even a minimal contribution may impact on the outcome of the smoking cessation. However, it is important to emphasize that the intensive approach is the most appropriate and should be prioritized.

\section{The Role of the Physical Therapist in Smoking Intervention}

Although there is a consensus that all health care professionals should advise and encourage smoking cessation for all patients, one study showed that less than $40 \%$ of adult smokers received any type of counselling [21].

It is known that the harmful effects of cigarettes extend to several systems of the human organism, such as cardiovascular, neurological and musculoskeletal and can interfere directly in clinical practice of physical therapists $[10,22]$. Thus, smoking detection is essential in physical therapy patients to promote counselling and appropriate intervention [10].

In addition, research shows that people who present some kind of disability, who habitually attend physical therapy, have a higher prevalence of smoking, which can be exacerbated by the aggregation of other risk factors such as obesity and a tendency to a sedentary lifestyle. Thus, the role of the physical therapist in working with the target population with a high prevalence of smoking can be observed [23].

Hence, the physical therapist presents particularities that allow a wide role in smoking intervention, such as frequent contact with the patient for extended periods and generally in an individualized manner. In other words, the physical therapy usually takes place several times a week for sessions of one hour or more and for longer periods of follow-up (months or years), characteristics specific to these professionals. When observing the activities of other health professionals, it is difficult to find such frequent contact with patients $[10,11]$.

It is worth mentioning that physical therapists are resident in various health centers and clinics, basic health units and hospitals, and thus, with knowledge of the different types of approach they could perform the smoking cessation procedures in all these environments. Therefore, these characteristics constitute an easy approach for smokers, regardless of why the patient is undergoing physical therapy $[10,24]$.

Physiotherapists play a key role in the multidisciplinary team. In practice, they provide secondary and tertiary prevention and can complement actions for mental health by associating health education and guidance with increasing levels of physical activity. Importantly, physical therapists, as well as other health professionals, should foster assistance and care for the prevention of diseases [24].

Contributing to smoking cessation may be considered an action for this purpose since all areas of operation of physical therapy benefit from smoking cessation as smoking affects multiple systems in the body [22].

\section{Effects of Tobacco on Other Systems and Its Influence in Physical Therapy}

As previously mentioned, smoking can influence and promote harmful effects in several systems of the human organism. Thus, these 
mechanisms and their correlations with interference in physical therapy in various areas need to be emphasized.

\section{Physical therapy in pulmonology}

The respiratory system is heavily damaged by smoking. The inhalation of cigarette smoke causes direct damage to the mucociliary transport, which is considered the main respiratory defense mechanism, possibly causing a greater chance of respiratory tract infections $[25,26]$. The high rate of respiratory symptoms and lung function decline related to smoking are also noteworthy [27].

Smoking is well established in the literature as the main cause of various lung diseases, including chronic obstructive pulmonary disease (COPD) [28]. After smoking, inflammatory cells, such as macrophages and leukocytes, migrate in large quantities to the lung and produce an inflammatory reaction that, over the years, has consequences on the lung structure and function. The obstruction mechanism is caused by several factors such as alterations in the quality and quantity of mucus, alterations in the small airways and loss of elastic recoil of the lungs [29].

In terms of the cardiopulmonary rehabilitation, it is common to see patients who continue smoking even after serious cardiac and pulmonary events. In this respect, it is essential to address the smoker, clarifying the harmful effects and influence of the components of cigarettes on physical therapy, as well as the high risk of recurrence of events in these systems. It is worth noting that the physical therapist is a professional with intense contact with patients in the context of cardiopulmonary rehabilitation [30]. Therefore, the conduct of the respiratory physical therapist with the smoker should take into account these alterations and consider techniques to reduce the clinical symptoms.

\section{Physical therapy in cardiology}

Tobacco use is one of the main risk factors for development of cardiovascular diseases and there are many effects of smoking on this system [31].

There is diverse evidence to demonstrate that smoking promotes coronary atherosclerosis and ischemic diseases through the arterial endothelial injury process, alteration in the lipid profile, reduction in vasodilator function and an increase in systemic inflammation levels $[32,33]$.

Moreover, smoking causes the release of catecholamine's that cause an increase in heart rate after smoking and promote long-term worsening of the responses of the autonomic nervous system, even during and after exercise. These factors may be related to arrhythmias and sudden death $[34,35]$.

Based on the above considerations, the physical therapist working in this area should remain alert to levels of heart rate, blood pressure and inadequate physiological responses during their practice.

It should also be mentioned that physical therapy plays a large role in the pre and postoperative periods of cardiovascular surgery; in this regard, smoking cessation becomes essential in order to avoid complications both in the preparation for surgery and recovery in the postoperative period. Thus, the patient in physical therapy in this area should be warned of the damage triggered by smoking on the cardiovascular system, the high risk of recurrence of cardiac events and the impact of smoking during their treatment.

\section{Physical therapy in urogynecology}

It is known that the action of carbon monoxide in the body promotes extensive damage to tissue oxygenation, including the sexual organs, which are widely vascularized and compromised by the action of cigarettes. This alteration may promote sexual impotence and decreased sensitivity in these organs, directly impacting on the sex lives of smokers [36].

Women are more susceptible to tobacco addiction, hence, another important aspect to be addressed in this area is smoking during pregnancy, which causes damage to both the mother and the fetus, and is directly related to premature births, perinatal deaths and underweight fetuses. In addition, studies have shown the negative influence of smoking on the development of the fetal nervous system [37]. There is also evidence showing that smoking increases the risk of infertility and increases the chances of difficulty conceiving [36]. Therefore, it is essential to include educational steps in the treatment of pregnant smokers during physical therapy to clarify all harmful effects of smoking on female health.

\section{Physical therapy in neurology}

Nicotine reaches the brain quickly; this toxicity affects cerebral circulation and may be related to the incidence and aggravation of various types of dementia such as Alzheimer's disease, brain tumours and an increased risk of stroke. The toxic effects of smoking may interfere directly in physical therapy given the fact that the consequences of continued tobacco use in the nervous system include: decline in language processing, impairment in visual and verbal memory, difficulties in learning and global motor function and even brain atrophy. Finally it should be noted that this toxin release mechanism promotes degeneration and interferes with the action of axons that are responsible for the conduction of nerve impulses, affecting the proper functioning of this system $[38,39]$.

Thus, the role of the physical therapist in advising the smoker is very important to minimize impairments and optimize the therapy in this area.

\section{Physical therapy in orthopaedics and sports}

The various toxic substances in cigarettes promote significant oxidative damage to important cellular structures. This oxidative stress process reaches the mitochondria and muscle proteins causing damage to muscle function. In addition, the regular use of tobacco promotes activation of inflammatory cells, such as interlecucina-6, C-reactive protein and tumor necrosis factor alpha, in several tissues [40,41].

Another important alteration that directly affects the muscular system is the increase of circulating carboxyhemoglobin, due to exposure to carbon monoxide, a major component of cigarette smoke. This process triggers a significant decrease in tissue oxygenation which can lead to increased fatigue and decreased muscle strength [42].

The skeletal system also presents alterations in smokers; studies show that smoking promotes bone demineralization which may cause a greater chance of fractures. This damage is attributable to alterations in the absorption of vitamin $\mathrm{D}$, high cortisol levels and decreased osteoclast activity; these associated factors reduce bone density, increasing the risk of fractures [43].

In accordance with the above, professionals involved in this area should be aware that physically active individuals and athletes can 
present significant losses in performance if they use tobacco $[44,45]$. Therefore, the alterations demonstrated in this particular area are of extreme importance as a major physical therapy treatment tool is physical exercise and its consequential effects on the musculoskeletal system.

\section{Smoker Intervention: Professional Role of the Physical Therapist}

Regardless of the reason that the patient is undergoing physical therapy, the approach to the smoker should be performed with care and empathy. It is important to provide guidance to the patient on the harmful effects of smoking. However, the focus of this approach should be the benefits that the individual can achieve by abstaining from the habit. It is possible to emphasize the short, medium and long term effects, such as the improvement in levels of blood pressure and heart rate, reduction in carbon monoxide levels, improved breathing and circulation, reduction in the risk of various heart and lung diseases, improvements in bad breath and stained teeth, the increase in exercise capacity and improvement in quality of life in general. The smoking cessation process is long and slow, so the health professional must constantly encourage and guide patients who choose to give up smoking [8].

The principle of smoking intervention should be to focus on behavioral change, since there is a very present behavioral mechanism in tobacco addiction. The initial guidance given to patients who decide to try cessation is to pick a date to quit smoking, after which this patient should be referred to a medical professional for prescription of medication assistance and ultimately the patient requires strategies to handle the symptoms during withdrawal, for example, keeping the mouth and hands busy with mints or chewing gum with a characteristic taste (ginger, cinnamon, mint, clove etc.) or peeling fruit and drinking lots of cold water. The principle of these strategies is rooted in the oral and manual reward to which the smoker is adapted, so that with the use of these guidelines there is a reduction in the need to smoke [8].

Other guidelines that need to be passed on to the smoker are: avoiding having any cigarettes stored at home, paying attention to food composition (avoiding high calorie foods, such as fried foods and sweets), taking medication properly, gaining weight cautiously and, moreover, including physical activity in the routine $[8,12]$.

Coupled with these recommendations, it is important that the professional detect risk situations that can lead to relapse, such as being close to people who smoke, not following proper prescription medication, consuming alcoholic drinks and coffee in the early days of termination (considered as triggers for cigarette cravings), having cigarettes in the home and not following the recommended guidelines $[12,13]$.

Breathing exercises and muscle relaxation should also be prioritized in the initial phase of smoking cessation, as they help to control the nervous crises, anxiety and irritability, very present in the initial phase of treatment [46].

All these strategies may be developed in any type of approach in which the physical therapist will act from the brief to the intensive, depending on the situation of each professional. It is important to emphasize that all types of counselling have an impact on the outcome of cessation and knowing the proper guidelines and the types of treatment is critical for all health professionals. However, if possible, intensive counselling is the most appropriate, since it demonstrates greater effectiveness in terms of cessation rates [47].

Another important strategy that can be directed by the physical therapist is physical activity. This should be considered and prescribed individually, when possible, to meet the needs of each smoker. Aerobic exercises that take longer to perform and require continuous oxygen consumption are interesting for those who suffer from cravings and other symptoms of withdrawal syndrome, since attention to somatic signs during exercise presents a unique strategy to distract smokers from anxieties and negative cognitions experienced during abstinence from smoking [48].

In addition to the potential benefits of exercise to moderate the withdrawal symptoms, exercise contributes positively to reducing the weight gain that patients often present after cessation [49] and there is evidence that it helps to reduce the craving for sweet foods during the first week of abstinence [50].

Exercise has also been shown to have a positive effect on other factors, seen as protective against relapse, including an increased ability to face challenges and increased self-esteem [51]. Additionally, being physically active has many benefits for overall health for both abstinent individuals and those who continue to smoke [52].

Thus the physical therapist together with the smoker can draw up plan of action for the entire termination process. The physical therapist should continually guide and give attention to the patient during this delicate moment, always asking about symptoms of the withdrawal syndrome and intensity of cravings while reinforcing that these symptoms are expected and short lived and reiterating the benefits that the patient will receive after tobacco cessation.

\section{Conclusion}

In conclusion, smoking remains a major public health problem throughout the world and health professionals prepared to work in smoking interventions are still scarce. Thus, the physical therapist through presenting unique particularities and characteristics can become facilitators in the smoking intervention process and, therefore, act both preventively and as interventionists in the smoking cessation process.

It is important that this professional become aware of their role in smoking cessation and act more clearly in this process, in order to optimize the treatment and prevent complications, seeking improvement in the health of their patients.

\section{Acknowledgements}

The authors would like to thank the Foundation of the State of São Paulo (FAPESP).

\section{References}

1. Pinto M, Ugá MAD (2010) The costs of tobacco-related diseases for the Unified Health System. Cad Saúde Pública 26: 1234-1245.

2. Saleheen D, Zhao W, Rasheed A (2014) Epidemiology and public health policy of tobacco use and cardiovascular disorders in low- and middleincome countries. Arterioscler Thromb Vasc Biol 34: 1811-1819.

3. World Health Organization (2008) Report on the global tobacco epidemic 2008: The MPower Package. Geneve: WHO.

4. Cavalcante TM (2005) The control of smoking in Brazil: advances and challenges. Rev Psiq Clin 32: 283-300. 
5. Silva ST, Martins MC, Faria FR, Cotta RMM (2014) Combating smoking in Brazil: The strategic importance of government actions. Cien Saude Colet 19: 539-552.

6. Doll R, Peto R, Boreham J, Sutherland I (2004) Mortality in relation to smoking: 50 years observations on male British doctors. BMJ 328: 1519

7. Godoy I (2010) Prevalence of smoking in Brazil: additional measures to control the disease should be prioritized in the Year of the Lung. J Bras Pneumol 36: 4-5.

8. Reichert J, Araújo AJ, Gonçalves CMC, Godoy I, Chatkin JM, et al. (2008) Guidelines for Smoking Cessation - 2008. J Bras Pneumol 34: 845-880.

9. Hartmann-Boyce J, Stead LF, Cahill K, Lancaster T (2014) Efficacy of interventions to combat tobacco addiction: Cochrane update of 2013 reviews. Addiction 109: 1414-1425.

10. Pignataro RM, Ohtake PJ, Swisher A, Dino G (2012) The role of physical therapists in smoking cessation: Opportunities for improving treatment outcomes. Phys Ther 92: 757-766.

11. Souza PS, Moreira J, Victor EG, Caretta LB, Tuon L (2013) Health education in the smoking control groups in the city of criciúma: the practice of the physiotherapist. Rev Health Public 6: 8-20.

12. Bodner ME, Rhodes RE, Miller WC, Dean E (2012) Smoking cessation and counselling practices of canadian physical therapists. Am J Prev Med 43:67-71.

13. Zhu SH, Lee M, Zhuang YL, Gamst A, Wolfson T (2012) Interventions to increase smoking cessation at the population level: How much progress has been made in the last two decades? Tob Control 21: 110-118.

14. Benowitz NL (2010) Nicotine Addiction. N Engl J Med 362: 2295-303.

15. Menossi HS, Goudriaan AE, de Azevedo-Marques CP, Nicastri S, de Andrade AG, et al. (2013) Neural bases of pharmacological treatment of nicotine dependence - insights from functional brain imaging: a systematic review. CNS Drugs 27: 921-941.

16. Lessov-Schlaggar CN, Pergadia ML, Khroyan TV, Swan GE (2008) Genetics of nicotine dependence and pharmacotherapy. Biochem Pharmacol 75: 178-195.

17. Planeta CS, Cruz FC (2005) Neurophysiological bases of tobacco dependence. Rev Psiq Clín 32: 251-258.

18. Rondina SC, Gorayeb R, Botelho C (2007) Psychological characteristics associated with tobacco smoking behavior. J Bras Pneumol 33: 592-601.

19. Clinical Guidelines on Supplementary Health (2011) Brazilian Medical Association and National Agency of Supplementary Health. Smoking.

20. Guideline Update Panel, Liaisons, and Staff (2009) Treating tobacco use and dependence: 2008 update U.S. Public health service clinical practice guideline executive summary. Respir Care 53: 1217-1222.

21. Gollust SE, Schroeder SA, Warner KE (2008) Helping smokers quit: understanding the barriers to utlization of smoking cessation services. Milbank Q 86: 601-627.

22. Nunes E (2006) Tobacco use and health effects. Rev Port Clin Geral 22:225-244.

23. Rimmer JH, Rowland JL (2008) Health promotion for people with disabilities: Implications for empowering the persona and promoting disability-friendly environments. Am J Lifestyle Med. 2008: 1-12.

24. Stephens JL, Lowman JD, Graham CL, Morris DM, Kohler CL, et al. (2013) Improving the validity and reliability of a health promotion survey for physical therapists. Cardiopulm Phys Ther J. 24: 14-23.

25. Proença M, Fagundes Xavier R, Ramos D, Cavalheri V, Pitta F, et al. (2011) Immediate and short term effects of smoking on nasal mucociliary clearance in smokers. Rev Port Pneumol. 17: 172-176.

26. Baby MK, Muthu PK, Johnson P, Kannan S (2014) Effect of cigarette smoking on nasal mucociliary clearance: A comparative analysis using saccharin test. Lung India. 31: 39-42.

27. Kotaki K, Senjyu H, Tanaka T, Yano Y, Miyamoto N, Nishinakagawa T, et al. (2014) Tobacco use among designated air pollution victims and its association with lung function and respiratory symptoms: a retrospective cross-sectional study. BMJ Open 31: 4: e005393.
28. From the Global Strategy for the Diagnosis, Management and Prevention of COPD, Global Initiative for Chronic Obstructive Lung Disease (GOLD) 2014

29. Kim JH, Park SG, Lee KH, Choi JH, Ha EH, et al. (2006) GSTM1 and GSTP1 Polymorphisms as potential factors for modifying the effect of smoking on inflammatory response. J Korean Med Sci 21: 1021-1027.

30. Collins EG, Bauldoff G, Carlin B, Crouch R, Emery CF, et al. (2014) Clinical Competency Guidelines for Pulmonary Rehabilitation Professionals: Position statement of the american association of cardiovascular and pulmonary rehabilitation. J Cardiopulm Rehabil Prev. 34: 291-302.

31. Anthony D, George P, Eaton CB (2014) Cardiac risk factors: Environmental, sociodemographic, and behavioral cardiovascular risk factors. FP Essent 421: 16-20

32. Ambrose JA, Barua RS (2004) The pathophysiology of cigarette smoking and cardiovascular disease: an update. J Am Coll Cardiol.43: 1731-1737.

33. Messner B, Bernhard D (2014) Smoking and cardiovascular disease: Mechanisms of endothelial dysfunction and early atherogenesis. Arterioscler Thromb Vasc Biol 34: 509-515.

34. Manzano BM, Vanderlei LC, Ramos EM, Ramos D (2011) Acute effects of smoking on autonomic modulation: analysis by Poincaré plot. Arq Bras Cardiol. 96: 154-160

35. Valenti VE, Vanderlei LC, Ferreira C, Fonseca FL, Oliveira FR, et al. (2013) Sidestream cigarette smoke and cardiac autonomic regulation. Int Arch Med 6:11.

36. British Medical Association, Board of Science and Education \& Tobacco Control Resource Centre (2004). Smoking and reproductive life: The impact of smoking on sexual, reproductive and child health. London: British Medical Association.

37. Leopércio W, Gigliotti A (2004) Smoking and its peculiarities during gestation: a critical review. J Bras Pneumol 30: 176-185.

38. Swan GE, Lessov-Schlaggar CN (2007) The effects of tobacco smoke and nicotine on cognition and the brain. Neuropsychol Rev 17: 259-273.

39. Rusanen M, Kivipelto M, Quesenberry CP, Zhou J, Whitmer RA (2011) Heavy smoking in midlife and long-term risk of Alzheimer disease and vascular dementia. Arch Intern Med 171: 333-339.

40. Barreiro E, Peinado VI, Galdiz JB, Ferrer E, Marin-Corral J, et al. (2010) Cigarette smoke-induced oxidative stress: A role in chronic obstructive pulmonary disease skeletal muscle dysfunction. Am J Respir Crit Care Med 182: 477-488.

41. Woodell A, Rohrer B (2014) A mechanistic review of cigarette smoke and age-related macular degeneration. Adv Exp Med Biol 801: 301-307.

42. Wüst RC, Morse CI, de Haan A, Rittweger J, Jones DA, et al. (2008) Skeletal muscle properties and fatigue resistance in relation to smoking history. Eur J ApplPhysiol 104: 103-110.

43. Abate M, Vanni D, Pantalone A, Salini V (2013) Cigarette smoking and musculoskeletal disorders. Muscles Ligaments Tendons 3: 63-69.

44. Papathanasiou G, Georgakopoulos D, Georgoudis G, Spyropoulos P, Perrea D, et al. (2007) Effects of chronic smoking on exercise tolerance and on heart rate-systolic blood pressure product in young healthy adults. Eur J Cardiovasc Prev Rehabil 14: 646-652.

45. Martinsen M, Sundgot-Borgen J (2014) Adolescent elite athletes' cigarette smoking, use of snus, and alcohol. Scand J Med Sci Sports 24:439-446.

46. Shahab L, Sarkar BK, West R (2013) The acute effects of yogic breathing exercises on craving and withdrawal symptoms in abstaining smokers. Psychopharmacology 225: 875-882.

47. Abrantes AM, Bloom EL, Strong DR, Riebe D, Marcus BH, et al. (2014) A preliminary randomized controlled trial of a behavioral exercise intervention for smoking cessation. Nicotine Tob Res 16: 1094-1103.

48. Ussher MH, Taylor AH, Faulkner GE (2014) Exercise interventions for smoking cessation. Cochrane Database Syst Rev 8:CD002295.

49. Farley AC, Hajek P, Lycett D, Aveyard P (2012) Interventions for preventing weight gain after smoking cessation. Cochrane Database Syst Rev. 
Citation: Freire FAPC, Ramos D, de Alencar Silva BS, Leite MR, Uzeloto JS, et al. (2017) The Role of Professional Physical Therapists in Smoking Cessation: A Literature Update. Gen Med (Los Angeles) 5: 287. doi:10.4172/2327-5146.1000287

Page 7 of 7

50. Teo AA, Allen AM, Allen SS, Oncken C (2014) Impact of exercise on changing food cravings in postmenopausal women during smoking cessation. Proceedings of Society for Research on Nicotine and Tobacco Annual Conference, Seattle, USA.

51. Spence JC, McGannon KR, Poon P (2005) The effect of exercise on global self-esteem: a quantitative review. J Sport Exerc Psychol.27: 311-334.
52. Garber CE, Blissmer B, Deschenes MR, Franklin BA, Lamonte MJ, et al. (2011) Quantity and quality of exercise for developing and maintaining cardiorespiratory, musculoskeletal, and neuromotor fitness in apparently healthy adults: guidance for prescribing exercise. Med Sci Sports Exerc 43: 1334-1359. 